\title{
The effects of stellar winds of fast-rotating massive stars in the earliest phases of the chemical enrichment of the Galaxy
}

\author{
G. Cescutti ${ }^{1}$ and C. Chiappini ${ }^{2,3}$
}

\author{
${ }^{1}$ Sezione di Astronomia, Dipartimento di Fisica, Universitá di Trieste, via G.B. Tiepolo 11, 34131 Trieste, Italy \\ e-mail: cescutti@oats.inaf.it \\ 2 Observatoire de Genève, Université de Genève, 51 Chemin des Maillettes, 1290 Sauverny, Switzerland \\ e-mail: cristina.chiappini@unige.ch \\ 3 INAF - Osservatorio Astronomico di Trieste, via G.B. Tiepolo 11, 34131 Trieste, Italy
}

Received 15 January 2010 / Accepted 27 February 2010

\begin{abstract}
Aims. We use the growing data sets of very-metal-poor stars to study the impact of stellar winds of fast rotating massive stars on the chemical enrichment of the early Galaxy.

Methods. We use an inhomogeneous chemical evolution model for the Galactic halo to predict both the mean trend and scatter of $\mathrm{C} / \mathrm{O}$ and $\mathrm{N} / \mathrm{O}$. In one set of models, we assume that massive stars enrich the interstellar medium during both the stellar wind and supernovae phases. In the second set, we consider that in the earliest phases $\left(Z<10^{-8}\right)$, stars with masses above $40 M_{\odot}$ only enrich the interstellar medium via stellar winds, collapsing directly into black holes.

Results. We predict a larger scatter in the $\mathrm{C} / \mathrm{O}$ and $\mathrm{N} / \mathrm{O}$ ratios at low metallicities when allowing the more massive fast-rotating stars to contribute to the chemical enrichment only via stellar winds. The latter assumption, combined with the stochasticity in the star formation process in the primordial Galactic halo can explain the wide spread observed in the N/O and C/O ratios in normal very-metal-poor stars.

Conclusions. For chemical elements with stellar yields that depend strongly on initial mass (and rotation) such as $\mathrm{C}, \mathrm{N}$, and neutron capture elements, within the range of massive stars, a large scatter is expected once the stochastic enrichment of the early interstellar medium is taken into account. We also find that stellar winds of fast rotators mixed with interstellar medium gas are not enough to explain the large CNO enhancements found in most of the carbon-enhanced very-metal-poor stars. In particular, this is the case of the most metal-poor star known to date, HE 1327-2326, for which our models predict lower N enhancements than observed when assuming a mixture of stellar winds and interstellar medium. We suggest that these carbon-enhanced very metal-poor stars were formed from almost pure stellar wind material, without dilution with the pristine interstellar medium.
\end{abstract}

Key words. Galaxy: halo - Galaxy: evolution - stars: abundances - stars: evolution - stars: rotation nuclear reactions, nucleosynthesis, abundances

\section{Introduction}

The very metal-poor stars of the halo play a fundamental role in chemical evolution since, at metallicities below $[\mathrm{Fe} / \mathrm{H}] \approx-2.5$, only type II supernovae have had time to contribute to the interstellar medium (ISM) enrichment from which these stars formed, thus offering a way of constraining the nucleosynthesis in massive stars at low metallicities (e.g. Chiappini et al. 2005; François et al. 2004).

At present, several thousand of very metal-poor stars (hereinafter VMP), i.e. stars with metallicities below $[\mathrm{Fe} / \mathrm{H}]=-2$. is known (e.g. Cayrel et al. 2004; Christlieb \& Beers 2005; Masseron et al. 2009). The samples of VMP stars are expected to increase by about one order of magnitude in the next years thanks to surveys such as SDSS/SEGUE-2 and LAMOST (see Beers 2010). Larger samples of VMP stars with high-quality abundances measurements will play a fundamental role in constraining the stellar nucleosynthesis of the first generations of massive stars.

To date, around $20 \%$ of the VMP stars observed show unexpected large $\mathrm{C}$ and $\mathrm{N}$ enhancements with respect to solar. These stars are called carbon-enhanced metal-poor stars (CEMP). The VMP stars not showing such large $\mathrm{C}$ and $\mathrm{N}$ enhancements are called normal VMP stars (Cayrel et al. 2004; Spite et al. 2005, 2006). In the case of the two most iron-poor stars known to date, these overabundances can reach 100 to 10000 times the ratios found in the Sun (Frebel et al. 2005, 2008; Christlieb et al. 2002; Norris et al. 2007).

The CEMP stars are classified according to the presence or absence of s- and r-process elements. The peculiar abundance of those showing over-abundances of s-process elements are usually interpreted as caused by accretion from an asymptotic giant branch (AGB) companion (e.g. Masseron et al. 2009, and references therein). However, those without s-process element enhancements were most probably formed from enriched gas ejected by earlier generations of massive stars (CEMP-no).

The study of the chemical enrichment of the pristine Universe in CNO, the most abundant metals, is of particular interest. The CNO chemical enrichment could have had a important impact in shaping the early IMF as well as on the production of $\mathrm{Li}, \mathrm{Be}$, and $\mathrm{B}$ from spallation of $\mathrm{C}, \mathrm{N}$, and $\mathrm{O}$ atoms in the early Universe. Moreover, the existence of CEMP-no stars suggests the synthesis of CNO to have played an important role in the first stellar generations.

In standard nucleosynthesis (without rotation), $\mathrm{N}$ is essentially a secondary element in massive stars, and has both primary 
and secondary components in low- and intermediate-mass stars. Carbon is produced as a primary element both in massive and low-and-intermediate mass stars. Oxygen is synthesized as a primary element in massive stars. Most massive stars would end their lives as type II SNe, dispersing their chemical make-up into the interstellar medium (ISM). However, it is believed that the most massive stars would collapse directly into black holes without the ejection of a supernova, especially at very-low metallicities (Heger et al. 2003) ${ }^{1}$. In this case, massive stars would contribute to the chemical enrichment of the ISM only via stellar winds.

The stellar yields of CNO can be affected by mass loss and rotation $^{2}$. In particular, the $\mathrm{C}$ and $\mathrm{N}$ yields of intermediate-mass stars still suffer from severe uncertainties related not only to mass loss rates, but also to the difficulties in modeling transport mechanisms (such as dredge-up episodes). In fact, there is evidence of additional mixing processes not included in standard models, triggered by rotation, gravity waves, and thermohaline mixing (e.g. Charbonnel \& Talon 2005) in this mass range.

The measurements of $\mathrm{C}, \mathrm{N}$, and the $\mathrm{C}$-isotopic ratio in normal VMP stars (Spite et al. 2005, 2006) have already set strong constraints on the nucleosynthesis of these elements by the first generations of massive stars. In fact, until 2004 no conclusive data were available for nitrogen in metal-poor halo stars. This situation has improved with the First Stars ESO large program (Cayrel et al. 2004), which obtained CNO abundances for the first time for a sample of giants with metallicities below $[\mathrm{Fe} / \mathrm{H}]=$ - 2.5 (Spite et al. 2005, 2006 - see also Fabbian et al. 2009). It turned out that these VMP halo stars had N/O ratios around solar, suggestive of high levels of production of primary nitrogen in massive stars. Moreover a large true scatter (more than the uncertainties in the derived abundances) has been found for the measured N/O. A large scatter has been also found for neutron capture elements in the same stars. These findings contrast with the results for alpha elements, which instead presented striking homogeneous $[\alpha / \mathrm{Fe}]$ ratios.

In Chiappini et al. (2005 - hereinafter C05), the implications of the new CNO data from Spite et al. (2005) on our understanding of nitrogen enrichment in the Milky Way were investigated. By the time the latter paper was published, there was no set of stellar yields able to explain the very metal-poor data from Spite et al. (2005). C05 concluded that the only way to account for the new data was to assume that massive stars at low metallicity rotate fast enough to produce larger amounts of nitrogen. As shown by Meynet \& Maeder (2002), rotationally-induced mixing transports the $\mathrm{C}$ and $\mathrm{O}$ produced in the He-burning core into the H-burning shell, where they are transformed in primary ${ }^{13} \mathrm{C}$ and ${ }^{14} \mathrm{~N}$. The efficiency of this process increases when the initial mass and rotational velocity increase, and the metallicity decreases (but see Ekström et al. 2008). C05 predicted that massive stars born with metallicities below $Z=10^{-5}$ should produce a factor of 10 up to a few times 100 more nitrogen (depending on the stellar mass) than the values given by Meynet $\&$ Maeder (2002) for $Z=10^{-5}$ and for $v_{\text {ini }}^{\text {rot }}=300 \mathrm{~km} \mathrm{~s}^{-1}$. This prediction has been confirmed by subsequent stellar evolution models computed at very low metallicities (Hirschi 2007), and higher rotational velocities, which were found to produce much more $\mathrm{N}$ than in the models of Meynet \& Maeder (2002).

\footnotetext{
1 Another possibility is the formation of faint supernovae, with large quantities of mixing and fall back as suggested by Nomoto and coauthors (e.g. Iwamoto et al. 2005).

${ }^{2}$ Fast-rotating massive stars can trigger stellar winds even at very-low metallicities - see Meynet et al. (2008).
}

Chemical evolution models computed with the new stellar evolution predictions turned out to not only account for the high N/O in normal-VMP stars (Spite et al. 2005), but also for the C/O upturn and low ${ }^{12} \mathrm{C} /{ }^{13} \mathrm{C}$ ratios (Spite et al. 2006) at very low metallicities (Chiappini et al. 2006, 2008). However, the same models cannot account for the huge $\mathrm{CNO}$ enhancements observed in CEMP stars.

Finally, C05 suggested that if the stellar yields of $\mathrm{N}$ are strongly dependent on the rotational velocities, hence on the mass of very-metal-poor massive stars, it is possible to understand the apparently contradictory finding by Spite et al. (2005) of a large scatter in N/O and the almost complete lack of scatter in $[\alpha / \mathrm{Fe}]$ ratios found in the same very metal-poor halo stars (Cayrel et al. 2004). Although the observed scatter could be related to the distribution of stellar rotational velocities as a function of metallicity, that the neutron capture elements in the same stars also show a large scatter pointed to a strong variation in the stellar yields with the mass range of the stars responsible by the synthesis of these elements. Cescutti (2008) explains simultaneously the observed spread in the neutron capture elements and the lack of scatter in the alpha elements as being caused by the stochasticity in the formation of massive stars, combined with the fact that massive stars of different mass ranges are responsible for the synthesis of the different chemical elements, namely: only massive stars with masses between 12 and $30 M_{\odot}$ contribute to the neutron capture elements, whereas the whole mass range of massive stars (10 to $80 M_{\odot}$ ) contribute to the production of alpha elements.

In the present paper we study the CNO evolution in the early phases of the galactic formation by means of the inhomogeneous code developed by Cescutti (2008) with the aim of reproducing not only the mean trend in the chemical abundances of $\mathrm{CNO}$ as in Chiappini et al. (2006), but also the spread in these particular elements. We assume the spread to be created by the stochasticity in the formation of stars, as in Cescutti (2008). Our aim is to see whether the same process as invoked by Cescutti (2008) to explain the scatter observed in the $\mathrm{r}$ - and s- process elements in normal stars also applies to CNO for which a large scatter is also observed. We tested the hypothesis that in the case of $\mathrm{CNO}$ the scatter is created by the fact that the most massive stars in the early Universe $\left(Z<10^{-8}\right.$ and $\left.M>40 M_{\odot}\right)$ were fast rotators, which collapse directly into a black hole (e.g. Heger et al. 2003), but contribute to the chemical enrichment of the ISM via stellar winds (Hirschi 2007; Meynet et al. 2006). We also investigate whether the predicted scatter is enough to account for the existence of CEMP-no stars.

In Sect. 2 we introduce the observational data we have adopted in the present paper. In Sect. 3 we briefly present our inhomogeneous model for the Galactic halo. The adopted stellar nucleosynthesis is described in Sect. 4. Section 5 is devoted to comparing our model predictions to the new data set of metalpoor stars in the solar vicinity. Finally, a discussion of our results and our conclusions are presented in Sect. 6.

\section{Observational data}

We compare our theoretical predictions with the measured abundances in halo stars. We distinguish the halo stars in four classes according to their metallicities: the ultra metal-poor stars (UMP), $[\mathrm{Fe} / \mathrm{H}]<-4.0$; the extremely metal-poor stars (EMP), $[\mathrm{Fe} / \mathrm{H}]<-3.0$; the metal-poor stars $[\mathrm{Fe} / \mathrm{H}]<-1.0$; and the CEMP stars, $[\mathrm{Fe} / \mathrm{H}]<-1.0$ and $[\mathrm{C} / \mathrm{Fe}]>0.9$. Christlieb et al. $(2002,2004)$ measured the first UMP star with an $[\mathrm{Fe} / \mathrm{H}]=-5.3$ : HE 0107-5240. In Frebel et al. (2005) and Aoki et al. (2006), 
we find the analysis of the chemical enrichment present in HE 1327-2326, the most iron-poor star known to date with $[\mathrm{Fe} / \mathrm{H}]=-5.5$. Frebel et al. (2006) show the first determination from a newly obtained VLT/UVES spectrum of the oxygen abundance in HE 1327-2326. For these two stars, we used the determination of the $\mathrm{CNO}$ abundance with the correction to the 1D LTE abundances for 3D effects taken from Frebel et al. (2008) for HE 1327-2326 and Collet et al. (2006) for HE 0107-5240. Norris et al. (2007) measured the abundance of HE 0557-8402, which has an iron abundance of $[\mathrm{Fe} / \mathrm{H}]=-4.75$. For this star a measurement of the abundance of carbon is available, but only upper limits for $\mathrm{N}$ and $\mathrm{O}$, so we prefer not to use their data, except in the $[\mathrm{C} / \mathrm{Fe}] \mathrm{vs}$. $[\mathrm{Fe} / \mathrm{H}]$ plot.

We employed the data from Spite et al. $(2005,2006)$ for the chemical abundances of $\mathrm{C}$ and $\mathrm{N}$, selecting only the unmixed extremely metal-poor stars of the Galactic halo. These data are part of the Large Program "First Stars". For O, we took the results obtained by Cayrel et al. (2004) into account with the correction for stellar surface inhomogeneities from Nissen et al. (2002); with this correction, the ratio is smaller in 3-D than in the 1-D computations. We also used the chemical abundances of 9 EMP stars from the recent work by Lai et al. (2008), who analyzed a total number of 28 stars. We used only the stars for which the abundances for $\mathrm{C}, \mathrm{N}$, and $\mathrm{O}$ were measured.

For the metal-poor stars of the halo, we exploited the recent data of Fabbian et al. (2008), assuming non-LTE for the lines of $\mathrm{O}$ and $\mathrm{C}$ with an efficiency equal to 1 for the collisions with neutral $\mathrm{H}\left(S_{\mathrm{H}}\right)$. Unfortunately, they do not take the $\mathrm{N}$ abundance into account: for this element in halo stars we used the data analyzed by Israelian et al. (2004) for (N/Fe) and N/O.

We also show the data for CEMP stars collected by Masseron et al. (2009). From their large data set of CEMP stars, we adopted only the data for stars with measured abundances of $\mathrm{C}, \mathrm{N}$, and $\mathrm{O}$ simultaneously. Moreover, we only considered stars with $[\mathrm{Ba} / \mathrm{Fe}]<1$ (CEMP-no) or $[\mathrm{Ba} / \mathrm{Eu}]<0$ (CEMP-r). We excluded stars with high abundances of s-process elements (CEMP-s), for which the most likely explanation seems to be pollution from a companion star.

\section{The chemical evolution model for the Milky Way halo}

The chemical evolution model we use in this work is based on the inhomogeneous model developed by Cescutti (2008) and on the homogeneous model of Chiappini et al. (2006). We consider that the Galactic halo consists of $100^{3}$ noninteracting cubic regions, each with the same typical volume of $2.8 \times 10^{6} \mathrm{pc}^{3}$. In this way the surface of the volume, taken as the surface of the side of a cube, is $2 \times 10^{4} \mathrm{pc}^{2}\left(S_{\text {cube }}\right)$. The dimension of the volume is large enough to allow us to neglect the interaction among different volumes and small enough to avoid losing the stochastic effects we are looking for. In fact, as we test for increasing volumes, the model tends to the homogeneous one (for details see Cescutti 2008). We use time steps of $1 \mathrm{Myr}$, which is shorter than any stellar lifetime considered here; in fact, the star with the maximum mass considered in the present work is $80 M_{\odot}$, and its lifetime is $\sim 3 \mathrm{Myr}$. At the same time, this time step is longer than the cooling time of the supernovae remnant bubbles, which is normally $\sim 0.1-0.2 \mathrm{Myr}$ and $0.8 \mathrm{Myr}$ at maximum (for details see Cescutti 2008).

\footnotetext{
3 We tested a larger number of volumes, and find that our results converge after around 100 volumes, although increasing considerably the computational time.
}

We choose to use parameters of the chemical evolution (star formation rate, initial mass function, stellar lifetime, nucleosynthesis) that are very similar to those of the homogeneous model by Chiappini et al. (2006). For this reason, the model is slightly different from the original inhomogeneous model by Cescutti (2008), and it improves the agreement with the metallicity distribution function of the Galactic halo.

In each region, we assume a Gaussian function as infall law of the gas with a primordial chemical composition:

$$
\frac{\mathrm{dGas}_{\text {Infall }}(t)}{\mathrm{d} t}=A \mathrm{e}^{\frac{-\left(t-t_{0}\right)^{2}}{2 \sigma^{2}}}
$$

where the parameter $A$ is equal to $1.28 \times 10^{4} M_{\odot} \mathrm{Myr}^{-1}, t_{0}$ is $250 \mathrm{Myr}$, and $\sigma$ is $100 \mathrm{Myr}$. As a result the formation of the halo takes place in $250 \mathrm{Myr}$. In each cube we assume a star formation rate $\psi(t)$, which is defined as:

$\psi(t)=v\left(\frac{M_{\mathrm{gas}}(t)}{S_{\text {cube }}}\right)^{1.5}$

where $M_{\text {gas }}(t)$ is the gas mass inside the considered box, and the parameter $v$ is $0.6 M_{\odot}^{-0.5} \mathrm{Myr}^{-1} \mathrm{pc}^{2}$. We we assume gas outflows to be proportional to $\psi(t)$, following the prescriptions of Chiappini et al. (2006):

$M_{\text {out }}(t)=f \cdot \psi(t)$

where $f=14$.

Knowing the mass that is transformed into stars in a time step (hereafter, $M_{\text {stars }}^{\text {new }}$ ), we assign the mass to one star with a random function, weighted according to an initial mass function (IMF). In this work, we show the results obtained by the model by adopting two different IMFs:

- Model 1 "standard" IMF of Scalo (1986) with two slopes:

$$
\phi(M)=C \cdot M^{-(1+x)} ; x=\left\{\begin{array}{l}
1.35 \text { if } 0.1 M_{\odot}<M<2.0 M_{\odot} \\
1.70 \text { if } 2.0 M_{\odot}<M<80.0 M_{\odot}
\end{array}\right.
$$

with $C=\frac{1}{\int_{0.1}^{80} M^{-x} d M}$.

- Model 2 "top heavy IMF" with the same slopes but with the mass range restricted between 1 and $80 M_{\odot}$ when $Z<1 \times$ $10^{-6}$ :

$$
\begin{aligned}
& \phi(M)=C^{\prime} \cdot M^{-(1+x)} ; x=1.70 \text { if } 1.0 M_{\odot}<M<80.0 M_{\odot} \\
& \text { with } C^{\prime}=\frac{1}{\int_{1}^{80} M^{-x} d M} .
\end{aligned}
$$

For $Z>1 \times 10^{-6}$ the normal mass range from 0.1 to $80 M_{\odot}$ is applied, assuming that around this critical metallicity the IMF switched to a normal one (see Schneider et al. 2002).

With the second IMF, we overcome the problem of having a high number of long-living metal free stars. Once the mass is assigned to the first star, we extract the mass of another star, and repeat this cycle until the total mass of newly-formed stars exceeds $M_{\text {stars }}^{\text {new }}$. In this way, in each region, and at each time step, the $M_{\text {stars }}^{\text {new }}$ is the same, but the total number and mass distribution of the stars are different, and we know the mass of each star contained in each region, when it is born, and when it will die, assuming the stellar lifetimes of Maeder \& Meynet (1989).

We compute the chemical evolution in the following way: at the end of its lifetime, each star enriches the interstellar medium with newly-produced elements (see the next section) as a function of its mass and metallicity. The total mass of each element 
is determined at the end of the lifetime of each star, by taking both the newly formed and the preexisting chemical elements into account.

The model does not take the pollution produced by stars with mass $<3 M_{\odot}$ into account because their lifetimes exceed the duration of the simulation. The existence of SNe Ia is also taken into account, according to the prescriptions of Matteucci \& Greggio (1986), in the single degenerate scenario. In fact, it is worth noting that the first type Ia SNe occur after only $30 \mathrm{Myr}$, the lifetime of an $8 M_{\odot}$ star (see Matteucci \& Recchi 2001 for a discussion on this point). With this model we are not only able to study the impact of different sets of stellar yields in the chemical enrichment of the Galactic halo, but also predict the expected spread for different chemical elements at low metallicities, where the random effects in the birth of stellar masses are important.

\section{Nucleosynthesis prescriptions}

In this section, we describe the set of the yields we adopt. The chemical elements we investigate are $\mathrm{CNO}$ and Fe. For the CNO yields, we use metal dependent yields for both lowand intermediate-mass stars and massive stars. For the low intermediate-mass stars, we adopt the stellar yields by Meynet $\&$ Meader (2002) with rotation for three metallicities, namely $Z=0.02, Z=0.004$, and $Z=10^{-5}$. We assume that their table for $Z=10^{-5}$ is valid down to $Z=0$, as in Chiappini et al. (2003). The table for yields of solar metallicity are published only in Chiappini et al. (2003). For the massive stars, we again assume the set of the yields by Meynet \& Meader (2002) for the three metallicities described above. We adopt two different sets of yields for $Z=10^{-8}$ :

- Model $a$ : In the first set of yields, we consider the ejected chemical elements at the explosion of the star as SNII (as in Chiappini et al. 2006), hereinafter named "total yields", because they are obtained as the sum the contributions during both the stellar wind and supernovae phases.

- Model $b$ : In the second set, we distinguish the yields for stars more massive and less massive than $40 M_{\odot}$. For the more massive stars, we only consider the chemical enrichment coming from the stellar wind, before the star collapses directly into a black hole (see Heger et al. 2003). We call this set of yields "wind yields". For the stars less massive than $40 M_{\odot}$ we again use the ejected chemical elements at the explosion of SNII.

The difference between total yields and wind yields is only in the yields for very massive stars $\left(M>40 M_{\odot}\right)$ and at very low metallicity, $Z=10^{-8}$. Both the "total" yields and the "wind" yields are taken from Hirschi (2007), when considering stars with very high rotational velocity (600 to $800 \mathrm{~km} \mathrm{~s}^{-1}$ ).

For iron, we adopt the solar metallicity yields of Woosley \& Weaver (1995) for $Z>10^{-8}$, whereas for $Z<10^{-8}$ we adopt the yields of Limongi (private communication), and the adopted yields of iron are shown in Fig. 1. These computations predict that population III stars with masses above $20 M_{\odot}$ inject negligible amounts of iron into the ISM upon their death. This agrees with the idea that the more massive pristine stars will collapse directly into black holes (Heger et al. 2003).

\section{Results}

We test 4 different models (Models A, B, C, and D) with different nucleosynthesis prescriptions and IMFs. The adopted

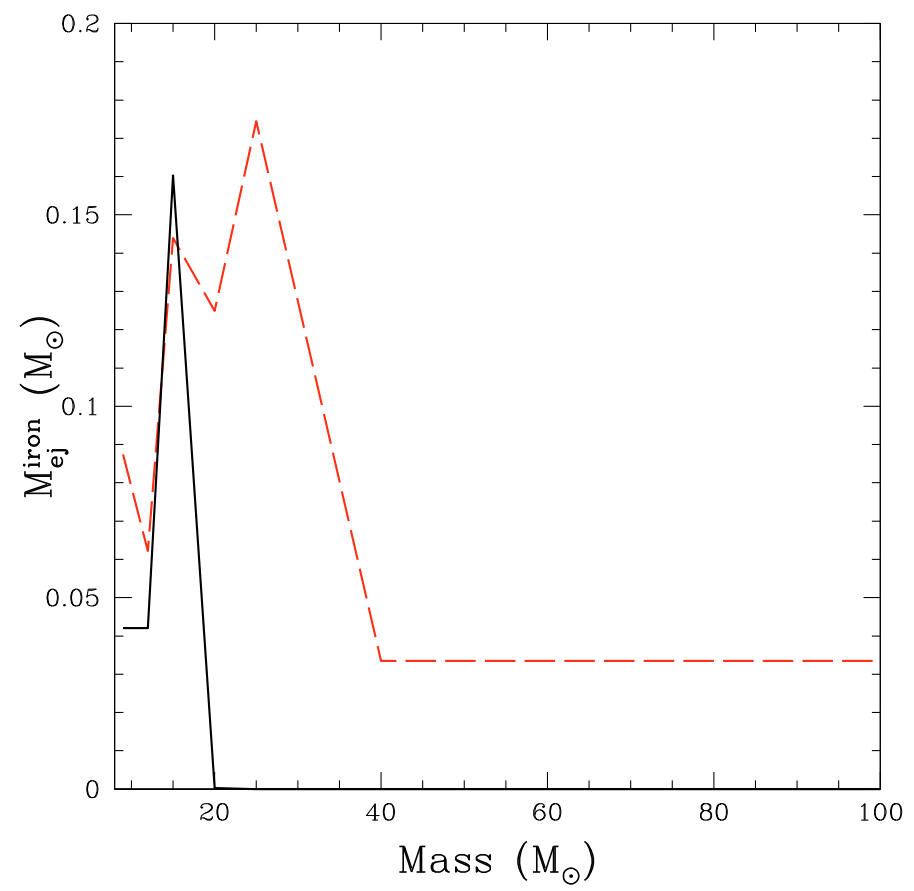

Fig. 1. Yields for iron computed by Limongi for $0<Z<10^{-8}$ (solid line), compared to the yields computed by WW95 at solar metallicity (dashed line).

Table 1. The nucleosynthesis prescriptions and IMFs adopted for the 4 models.

\begin{tabular}{ccc}
\hline \hline Model & IMF & yields \\
\hline A & "top heavy" (Model 2) & "total yields" (Model a) \\
B & "top heavy" (Model 2) & "wind yields" (Model b) \\
C & "standard" (Model 1) & "total yields" (Model a) \\
D & "standard " (Model 1) & "wind yields" (Model b) \\
\hline
\end{tabular}

combinations of Models 1 and 2 (different IMFs) and Models a and $b$ (different set of stellar yields) are summarized in Table 1.

In the figures that follow we only plot the abundance ratios of simulated stars still-living at the present time (i.e. stars with masses $<1 M_{\odot}$ ). In Fig. 2 we show the results of model A (blue) and $\mathrm{B}$ (red) for the ratio of $\log (\mathrm{N} / \mathrm{O})$ (top panel) and $\log (\mathrm{C} / \mathrm{O})$ (bottom panel). Both models can reproduce the observed abundance trend for halo stars in the range $6<\log \epsilon(\mathrm{O})<8$. Our models do not extend to the metallicity range of the galactic disk, as they are pure halo models. The different models show different behaviors at very low metallicity. At these earliest phases, in fact, the differences in the assumed yields are very important.

In model B, we assume that the very massive stars end their lives directly as black holes (see Heger et al. 2003), and only the newly produced elements ejected by these massive stars in the stellar wind phase enrich the ISM, as explained in Sect. 3. The calculations of Hirschi (2007) predict high N/O and C/O ratios in the stellar winds of fast-rotating massive stars, higher than what is predicted for their total yields (i.e. including the material ejected during the SNII explosion).

In Fig. 2 the stochasticity of the code clearly shows its peculiarity, because it predicts that some of the stars observed today will have high $\mathrm{C} / \mathrm{O}$ and $\mathrm{N} / \mathrm{O}$ ratios (Model $\mathrm{B}$ ), but also predicts that some stars will have the same ratio as those predicted by Model A, which was computed with total yields for the whole mass range. This is expected because in Model B we adopted the wind yields only for the most massive stars. Such a result 
G. Cescutti and C. Chiappini: The effects of stellar winds of fast rotating massive stars

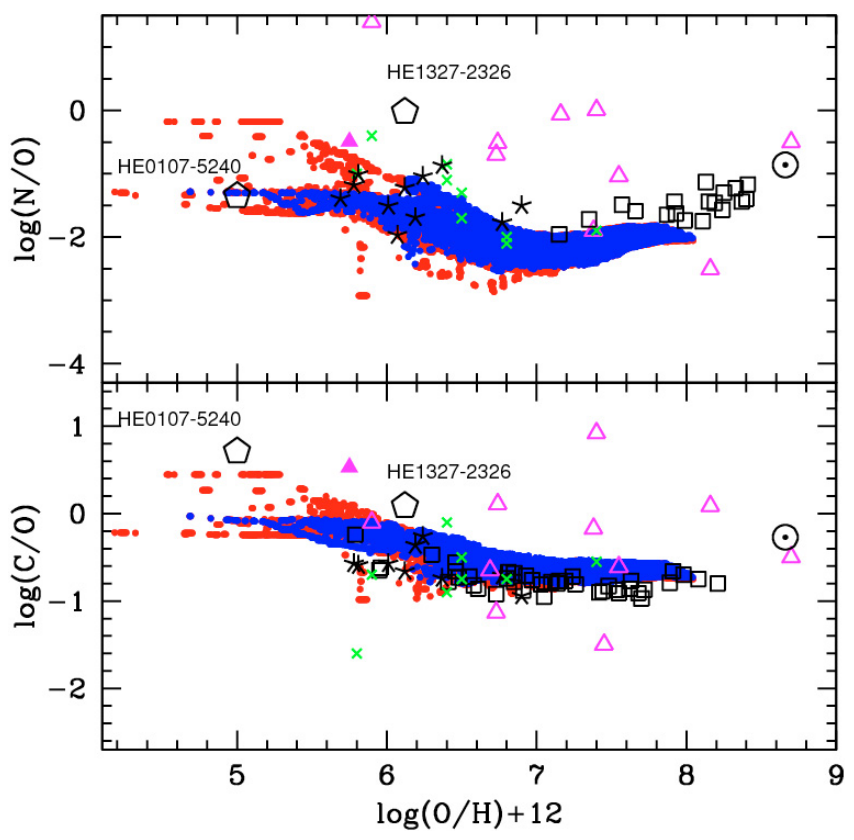

Fig. 2. $\log (\mathrm{N} / \mathrm{O})$ vs. $\log \epsilon(\mathrm{O})$ (upper panel), $\log (\mathrm{C} / \mathrm{O})$ vs. $\log \epsilon(\mathrm{O})$ (lower panel); the still-living simulated stars from model A are in blue and from model B in red. Starred symbols are for the data from Spite et al. (2006), crosses are for the data from Lai et al. (2008); the open square are data from Fabbian et al. (2009) for $\log (\mathrm{C} / \mathrm{O})$ and by Israelian et al. (2004) for $\log (\mathrm{N} / \mathrm{O})$. The large open pentagons are the UMP stars, for which we insert the name of the star close to the respective pentagon. In this plot we also show the data for CEMP-no stars with open triangles and for the CEMP-r star with a filled triangle. These data have been collected by Masseron et al. (2009). The symbols of the Sun refer to the the solar values measured by Asplund et al. (2005).

only can be obtained with an inhomogeneous model, since standard homogenous chemical models would instead predict a single trend representing the mean contribution of all the stars dying at a given metallicity. This is a very interesting and important feature of the chemical inhomogeneous model, and it represents the novelty of this approach.

The predictions of model B for $\mathrm{N}$ match the observed trend, and it is also able to explain the chemical abundances of the UMP star HE 0107-5240 (see also Figs. 6 and 7 where the results for $[\mathrm{C} / \mathrm{Fe}],[\mathrm{N} / \mathrm{Fe}]$, and $[\mathrm{O} / \mathrm{Fe}]$ are shown). The spread predicted by model $\mathrm{B}$ is a consequence of our hypothesis that massive stars have two different fates, with quite different nucleosynthesis at very low metallicities. This assumption seems to be crucial for explaining the observed scatter in the $(\mathrm{N} / \mathrm{O})$ ratio of normal stars.

However, this model is not able to explain the $\log (\mathrm{N} / \mathrm{O})$ of HE 1327-2326, predicting too low values for this ratio around 6.5 in $\log \epsilon(\mathrm{O})$. This suggests that HE 1327-2326 was essentially formed only from the wind material, without dilution with the pristine ISM material. The formation from the pure wind material of new stars is not taken into account in our chemical model. In our model the ejecta from stars, whether wind yields or total yields, are mixed and diluted with the whole ISM gas present in the simulated region.

In fact, this star shows low $\mathrm{Li}$ abundance, which is expected if the material from which this object formed was essentially pure stellar wind material where the primordial Li was destroyed. In addition, this star has a potentially low ${ }^{12} \mathrm{C} /{ }^{13} \mathrm{C}$ (as only a lower limit of 5 is given by Aoki et al. 2006) typical of hydrogen-burned material (see Meynet et al. 2010). We notice that HE0107-5240 has a ${ }^{12} \mathrm{C} /{ }^{13} \mathrm{C}=60 \pm 10$ (Christlieb, priv. commun.), compatible with what is expected from an ISM enriched by fast-rotating stars (see Chiappini et al. 2008).

Model A, computed with "total" yields for all massive stars, does not reproduce the data as well as model B. This is true not only for the UMP stars, but also for the EMPs, which show some objects with very high N/O ratios towards the lowest values of $\log \epsilon(\mathrm{O})$, which is not predicted by the models.

For C/O, the measurements of metal-poor normal stars (Spite et al. 2006) show much less spread than what is found for N/O. In fact, although stellar models with rotation also predict higher $\mathrm{C} / \mathrm{O}$, this increase is much less important than the one in $\mathrm{N}$. This means that the $\mathrm{N}$ yields are much more dependent on the initial rotational velocity, hence on the stellar mass, than the $\mathrm{C}$ yield. This explain the smaller scatter predicted for the $\mathrm{C} / \mathrm{O}$ than for the one of $\mathrm{N} / \mathrm{O}$.

On the other hand, none of the models is able to reproduce the abundances of the UMP stars, although model B marginally agrees with the C/O of both HE 1327-2326 and HE 0107-5240. Very interestingly, none of our models is able to reproduce the observed abundance ratios of the CEMP-no stars (see Fig. 2 for the $\mathrm{C} / \mathrm{O}$ and $\mathrm{N} / \mathrm{O}$ and Fig. 6 for the $\mathrm{C} / \mathrm{Fe}, \mathrm{N} / \mathrm{Fe}$, and $\mathrm{O} / \mathrm{Fe}$ ). We thus conclude that the chemical abundances of the CEMP-no stars cannot be explained in the same framework as the abundances of normal VMP stars. The effect of stellar winds of fast rotators plus the inhomogeneity of the ISM are not strong enough to predict the large observed enhancements in the CNO elements. As for the UMP stars discussed above (also CNO enhanced), the CEMP-no stars were probably formed from almost pure wind material. It would be very useful to better constrain our scenario to have the abundances of $\mathrm{Li}$ and ${ }^{13} \mathrm{C}$ for all CEMPno stars (see Meynet et al. 2010). The case of the only CEMP-r star for which we have the abundances of $\mathrm{C}, \mathrm{N}$, and $\mathrm{O}$ (represented by a filled triangle in Figs. 2 and 6) is rather similar to the other CEMP-no discussed above.

In Fig. 3 the predictions of models $\mathrm{C}$ and $\mathrm{D}$ (both models assume a standard IMF - see Table 1) are shown. The results are similar to the ones of models A and B. This suggests that it is not necessary to consider top-heavy IMF to explain the chemical abundances of CNO at low metallicity, once the contribution of fast rotators is taken into account.

However, models A and B, with a top-heavy IMF, do overcome the problem of producing a significative number of zero metallicity stars (still living) which have not been observed up to now. In addition, models $\mathrm{A}$ and $\mathrm{B}$ produce slightly more massive stars than models $\mathrm{C}$ and $\mathrm{D}$, as is barely visible when comparing Figs. 2 and 3. This can be seen more clearly in Fig. 4. This figure shows the distribution of the simulated stars with respect to the $\mathrm{C} / \mathrm{O}$, for the models $\mathrm{A}, \mathrm{B}, \mathrm{C}$, and $\mathrm{D}$. To better disentangle the differences at low metallicity we split the resulting stars in two ranges: $\log \epsilon(\mathrm{O})<5.5$ in the upper panel, $5.5<\log \epsilon(\mathrm{O})<6.5$ in the lower panel. In the upper panels, both models B (on the left) and $\mathrm{D}$ (on the right) show a peak at $\log (\mathrm{C} / \mathrm{O}) \sim 0$. On the right of this peak, there is another lower peak. This secondary peak is produced by stars formed in a volume where the ISM was enriched by the stellar winds of fast rotators, before fast rotators of lower mass have had time to eject their products via the supernovae phase. As the stellar winds of fast rotators are richer in C compared to what is ejected by the $\mathrm{SN}$ explosion, these volumes will produce stars with higher $\mathrm{C} / \mathrm{O}$. Most of the stars will in fact originate in a gas where the contamination of less massive stars dominated (i.e. which polluted the ISM with their total yields upon the explosion of the $\mathrm{SN}$ ). 


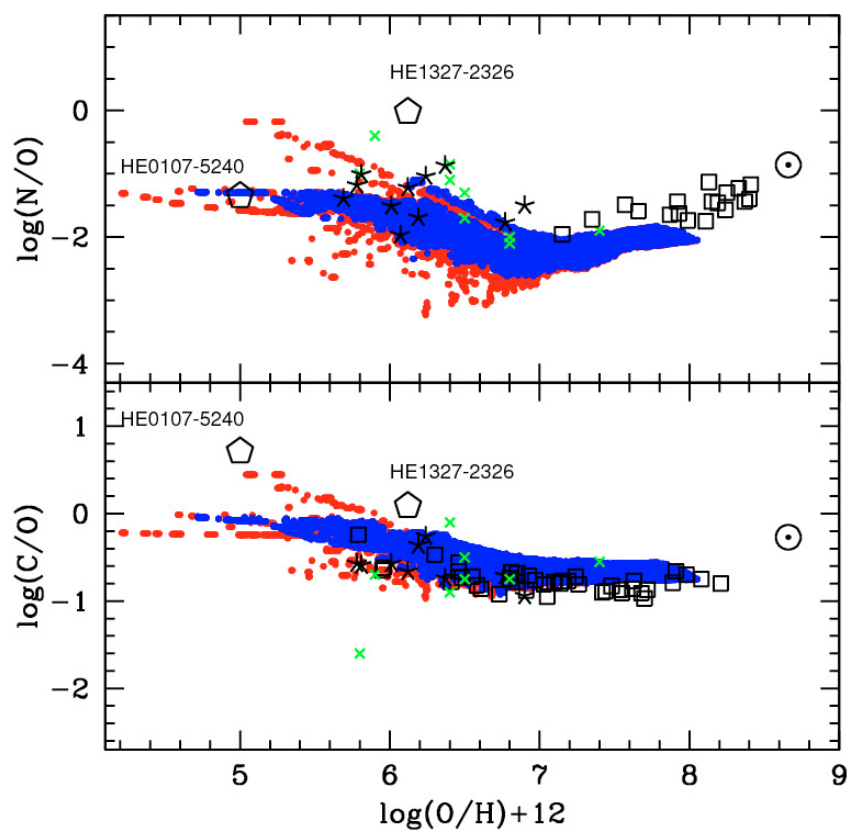

Fig. 3. $\log (\mathrm{N} / \mathrm{O})$ vs. $\log \epsilon(\mathrm{O})$ (upper panel), $\log (\mathrm{C} / \mathrm{O})$ vs. $\log \epsilon(\mathrm{O})$ (lower panel). The still-living simulated stars from model $\mathrm{C}$ are in blue and from model D in red. Starred symbols are for the data from Spite et al. (2006), crosses are for the data from Lai et al. (2008); the open square are data from Fabbian et al. (2009) for $\log (\mathrm{C} / \mathrm{O})$, and by Israelian et al. (2004) for $\log (\mathrm{N} / \mathrm{O})$. The large open pentagons are the UMP stars, for which we insert the name of the star close to the respective pentagon. The symbols of the Sun refer to the the solar values measured by Asplund et al. (2005).

The second peak for model B is slightly higher than the one of model D because of the different IMF used (see Table 1), creating more massive stars. In the lower panels we compare the results of our models in a range of metallicity slightly broader, with the distribution of the observed stars in the same range (we do not show the distribution of the observed stars in the upper panel because we only have the UMP star HE 0107-5240). For $\mathrm{C}$, in this metallicity range, the agreement between the observed distribution and what is predicted by model $\mathrm{B}$ is remarkable. In fact, our predictions agree not only with the peak value but also with the observed spread. We underline that the number of stars observed in this range is very low (15) and larger statistics are needed to better constrain our models. Model A reproduces the peak value too, but this model does not predict the observed spread; in particular, it does not produce stars with high values for $\mathrm{C} / \mathrm{O}$. Toward a low value for this ratio, both model $\mathrm{A}$ and $\mathrm{B}$ do not explain all the spread present in the observed stars, but again the model B predicts this feature slightly better. The results for models $\mathrm{C}$ and $\mathrm{D}$ are very similar to the ones of models $A$ and $B$, since the top-heavy IMF does not influence much at this stage, but model B still seems to reproduce the spread in the ratio of $\mathrm{C} / \mathrm{O}$ for the observed stars slightly better than model $\mathrm{D}$.

Our predictions for the distribution of N/O, for the four models are shown in Fig. 5. In the top panel, where the results are shown for $\log \epsilon(\mathrm{O})<5.5$, models computed with wind yields do not produce a secondary peak as for carbon, but rather a flat distribution towards higher $\log (\mathrm{N} / \mathrm{O})$ values. This is due to the different behavior of adopted yields for very massive stars in the set of the wind yields. Nevertheless, again model B compared to model D shows higher number of stars with an enhanced $\log (\mathrm{N} / \mathrm{O})$, again due to the different IMF adopted.

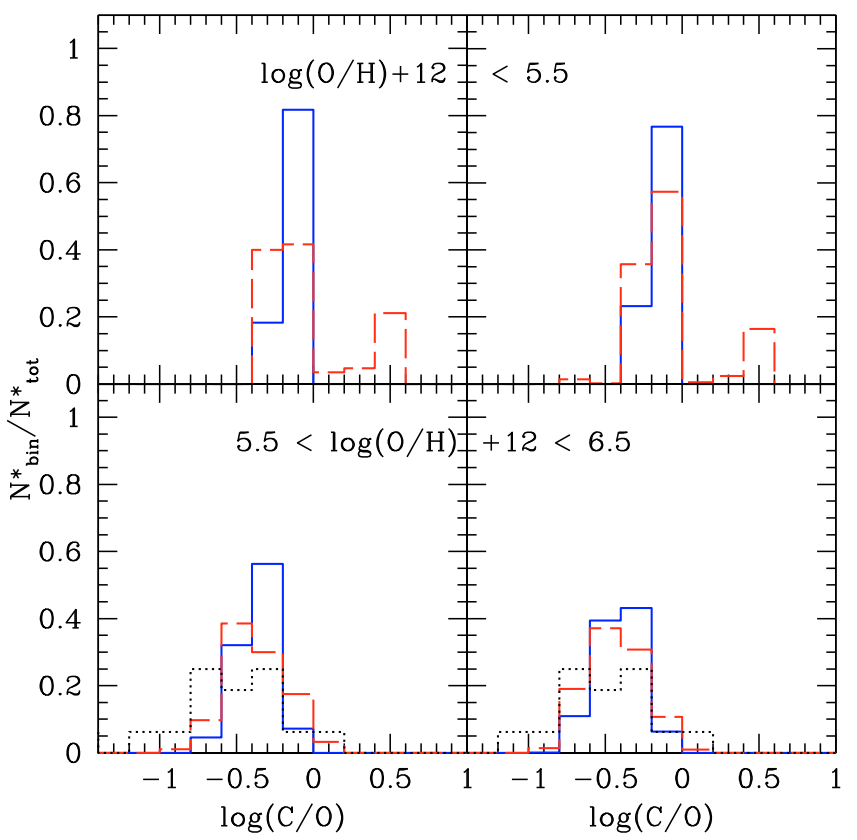

Fig. 4. In the left panels, the histograms for the distributions of simulated stars in bins of $\log (\mathrm{C} / \mathrm{O})$, for models A (blue solid) and B (red dashed). In the right panels, the histograms for the distributions of simulated stars in bins of $\log (\mathrm{C} / \mathrm{O})$ for models $\mathrm{C}$ (blue solid) and $\mathrm{D}$ (red dashed). In the upper panels stars with $\log \epsilon(\mathrm{O})<5.5$, in the lower panels stars with with $5.5<\log \epsilon(\mathrm{O})<6.5$. In the lower panels, the distribution of the observed normal metal-poor is also shown stars with a black dotted line.

In the lower panel for $5.5<\log \epsilon(\mathrm{O})<6.5$, we show the distribution for the observed stars, too. On the bottom left, it can be see that the predicted N/O by model B shows a spread that is very similar to the observed one but that predicts a ratio around 0.4 dex lower than the one indicated by the data. In the next figures, we show that the reason for this difference relies on the yields of $\mathrm{N}$ (rather than $\mathrm{O}$ ) in the $5.5<\log \epsilon(\mathrm{O})<6.5$ metallicity range. Model A presents a narrower spread for N/O than model B. Models C and D (bottom right) show similar results to models $\mathrm{A}$ and $\mathrm{B}$, respectively, at this metallicity range.

The results presented in Figs. 4 and 5 illustrate that inhomogeneous models can go beyond the interpretation of abundance ratio trends with metallicity, also predicting scatter at the different phases of the chemical enrichment history of the Galactic halo. At present, the poor statistics of the observations limit the utility of such models. Here a comparison between our theoretical models and observations is only possible in a very limited metallicity range $-5.5<\log \epsilon(\mathrm{O})<6.5-$, due to the lack of data. This situation will improve soon with the upcoming large datasets for halo stars (from surveys such as SEGUE-2 and LAMOST - see Beers 2010), which will play a fundamental role in constraining this kind of model.

Our predictions for $[\mathrm{C} / \mathrm{Fe}],[\mathrm{N} / \mathrm{Fe}]$, and $[\mathrm{O} / \mathrm{Fe}]$ are shown in Figs. 6 (models $\mathrm{A}$ and $\mathrm{B}$ ) and 7 (models $\mathrm{C}$ and D). In these plots we consider the solar abundances by Asplund et al. (2005). The results of the models in these plots are not completely self-consistent from the point of view of nucleosynthesis as the iron yields came from different authors who include the explosive nucleosynthesis, whereas the CNO yields come from the theoretical predictions of the Geneva group, which include only the pre-supernovae phase. Our models can reproduce these ratios well, and also predict some stars in the locus of HE 0107-5240, whereas HE 1327-2326 is not reproduced. 
G. Cescutti and C. Chiappini: The effects of stellar winds of fast rotating massive stars

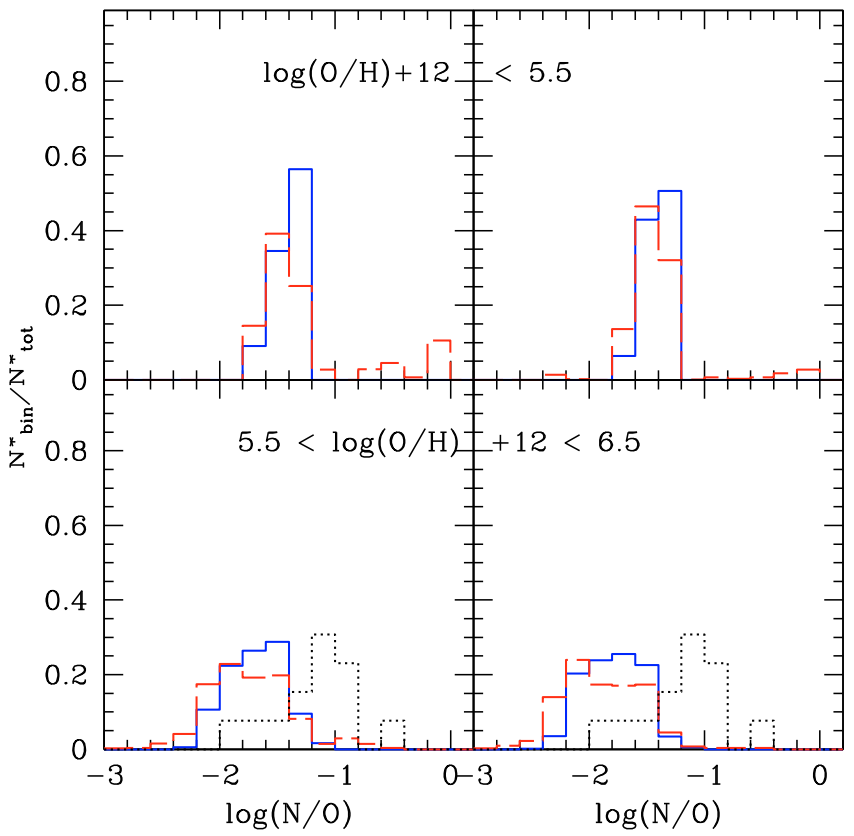

Fig. 5. In the left panels, the histograms for the distributions of simulated stars in bins of $\log (\mathrm{N} / \mathrm{O})$ for models A (blue solid) and B (red dashed). In the right panels, the histograms for the distributions of simulated stars in bins of $\log (\mathrm{N} / \mathrm{O})$ for models $\mathrm{C}$ (blue solid) and $\mathrm{D}$ (red dashed). In the upper panels we consider stars with $\log \epsilon(\mathrm{O})<5.5$, in the lower panels stars with with $5.5<\log \epsilon(\mathrm{O})<6.5$. In the lower panels, the distribution of the observed normal metal-poor stars is also shown with a black dotted line.

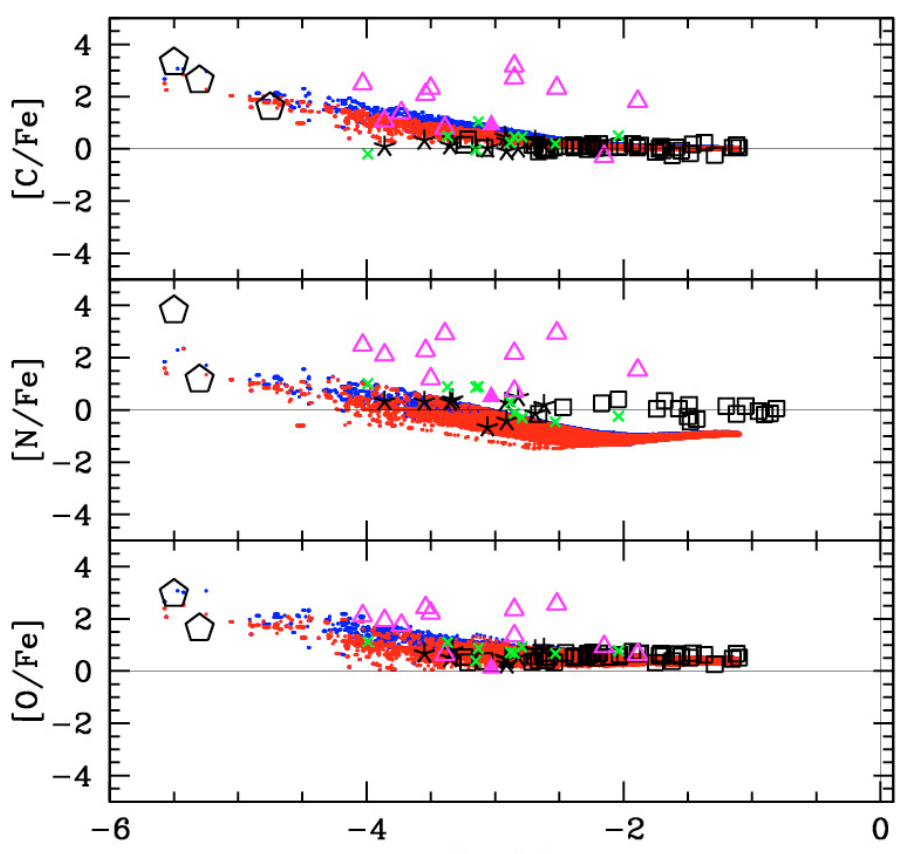

$[\mathrm{Fe} / \mathrm{H}]$

Fig. 6. $[\mathrm{C} / \mathrm{Fe}]$ vs. $[\mathrm{Fe} / \mathrm{H}]$ (upper panel), $[\mathrm{N} / \mathrm{Fe}]$ vs. $[\mathrm{Fe} / \mathrm{H}]$ (middle panel), $[\mathrm{O} / \mathrm{Fe}]$ vs. $[\mathrm{Fe} / \mathrm{H}]$ (lower panel). The still-living simulated stars for model A in blue and for model B in red, starred symbols are for the data from Spite et al. (2006), crosses are the data from Lai et al. (2008). The open squares are data from Israelian (2004) for $[\mathrm{N} / \mathrm{Fe}]$ and from Fabbian et al. (2009) for $[\mathrm{C} / \mathrm{Fe}]$ and $[\mathrm{O} / \mathrm{Fe}]$. The large pentagons are the UMP, described in the observational data. In this plot we also show the data for CEMP-no stars with open triangles and for the CEMP-r star with a filled triangle. These data have been collected by Masseron et al. (2009).

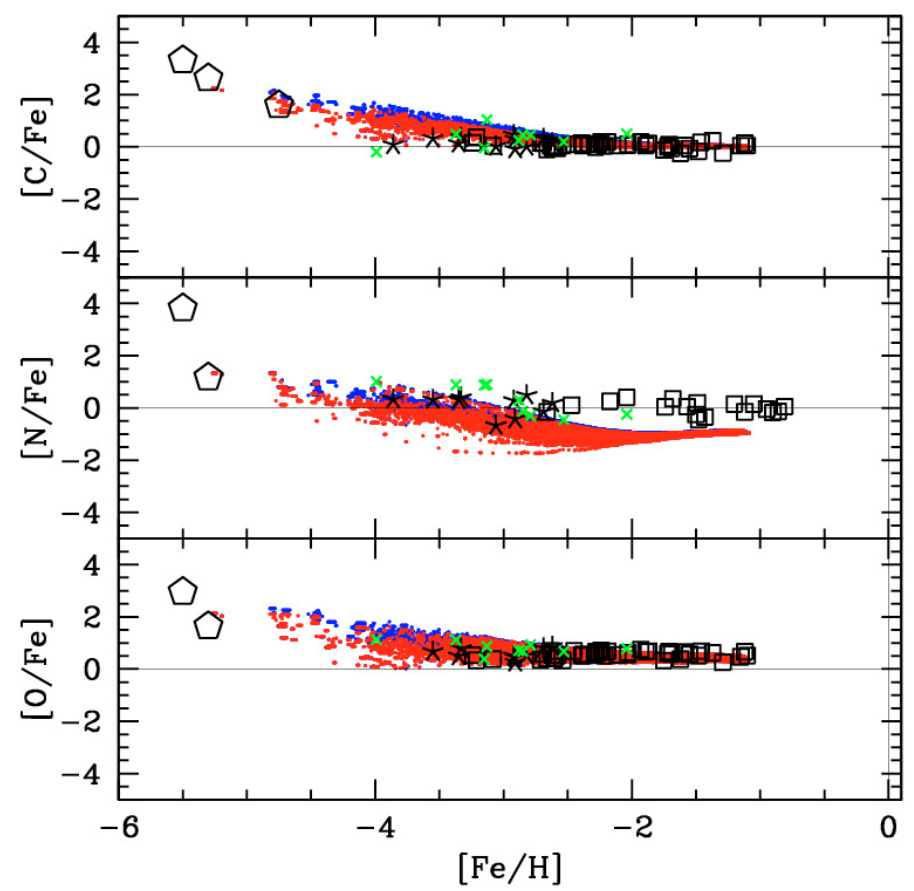

Fig. 7. $[\mathrm{C} / \mathrm{Fe}]$ vs. $[\mathrm{Fe} / \mathrm{H}]$ (upper panel), $[\mathrm{N} / \mathrm{Fe}]$ vs. $[\mathrm{Fe} / \mathrm{H}]$ (middle panel), $[\mathrm{O} / \mathrm{Fe}]$ vs. $[\mathrm{Fe} / \mathrm{H}]$ (lower panel). The still-living simulated stars for model $\mathrm{C}$ in blue and for model $\mathrm{D}$ in red, starred symbols are for the data from Spite et al. (2006), crosses are the data from Lai et al. (2008). The open squares are data from Israelian (2004) for $[\mathrm{N} / \mathrm{Fe}]$ and from Fabbian et al. (2009) for $[\mathrm{C} / \mathrm{Fe}]$ and $[\mathrm{O} / \mathrm{Fe}]$. The large pentagons are the UMP, described in the observational data.

Moreover, our models cannot account for the CEMP-no stars. For nitrogen, there is a marginal disagreement in the intermediate metallicity range, pointing to an extra source of nitrogen not accounted for in our models. As discussed in Chiappini et al. (2006, 2008) and Pipino et al. (2009), this is most probably because current chemical evolution models do not include the contribution of super AGB stars. Super AGB stars could contribute with important quantities of nitrogen, and their contribution would be visible just before the appearance of the intermediatemass stars (Chiappini et al., in prep.).

Finally, although our models cannot explain the $[\mathrm{N} / \mathrm{Fe}]$ ratio of HE 1327-2326, we are able to reach the very low enrichment in $\mathrm{Fe}$ observed in this star and to predict almost the correct ratios of $[\mathrm{C} / \mathrm{Fe}]$ and $[\mathrm{O} / \mathrm{Fe}]$ in models $\mathrm{A}$ and $\mathrm{B}$, in the chemical inhomogeneous framework. Moreover, we reproduce the ratio of the three elements $[\mathrm{C} / \mathrm{Fe}],[\mathrm{N} / \mathrm{Fe}]$, and $[\mathrm{O} / \mathrm{Fe}]$ for the other star with an $[\mathrm{Fe} / \mathrm{H}] \lesssim-5$, namely, $\mathrm{HE} 0107-5240$. We also reproduce HE 0557-4840 discovered and analyzed by Norris et al. (2007) for which we only have the $[\mathrm{C} / \mathrm{Fe}]$ ratio.

\section{Conclusions}

In this paper we have shown the results of an inhomogeneous chemical evolution model for the mean trend and scatter of $\mathrm{C} / \mathrm{O}$ and N/O in the Galactic halo, taking the contribution of fastrotator stars into account.

The observational studies of chemical enrichment in very metal-poor stars of the halo have found a large scatter (larger than the uncertainties in the derived abundances) for the measured $\mathrm{C} / \mathrm{O}$ and N/O. A large scatter was also found for neutron capture elements in the same stars. These findings contrast with the results for $\alpha$-elements, which instead presented striking 
homogeneous $[\alpha / \mathrm{Fe}]$ ratios. In Cescutti (2008), the spread in the chemical abundance ratios of neutron capture elements has been explained with a different mass range for the production of these elements (from 12 to $30 M_{\odot}$ ), compared to the whole range of massive stars for the $\alpha$-elements.

In this work the nucleosynthesis differences for the elements $\mathrm{C}, \mathrm{N}$, and $\mathrm{O}$ come from the rotation of massive stars, which strongly affects the ratio of the production among these elements at low metallicity (see Hirschi et al. 2007). Moreover, we consider two possible contributions to the enrichment of the ISM by massive stars at very low metallicity, the usual enrichment through supernovae ejecta, and the enrichment only through their stellar winds.

We find that the assumption that the most massive fast rotators only contribute to the ISM enrichment via stellar winds leads to chemical evolution models that are able to account for both the large scatter in the $\mathrm{N} / \mathrm{O}$ and $\mathrm{C} / \mathrm{O}$ and the simultaneous lack of scatter in $\alpha$-elements observed in very metal-poor normal halo stars. In fact, the stellar yields of the latter elements do not show any strong dependency on the stellar mass, contrary to what happens for $\mathrm{N}, \mathrm{C}$, and n-capture elements.

In this context, we also explored whether the scatter in $\mathrm{N}$ and $\mathrm{C}$ created by the strong yields dependence on the stellar mass, when including the fast rotators, would also account for the existence of the so-called carbon-enhanced stars (in particular the CEMP-no ones). We find that, even when considering that the more massive fast rotators enrich the early ISM mainly via stellar winds strongly enhanced in $\mathrm{CNO}$, it is impossible to explain the abundances observed in CEMP-no stars. However, we point out that the latter model marginally agrees with the abundance ratios of the only CEMP-r star for which the CNO abundances have been measured, and of the two of the three UMP stars known to date, HE 0107-5240 and HE 0557-4840. These results suggest that very metal-poor massive stars would collapse directly into black holes as predicted by Heger et al. (2003).

The discrepancy with respect to the CEMP-no stars, and in particular, HE 1327-2326, suggest that these objects are born from the gas expelled during the wind phase of fast rotators, without much mixing with the surrounding ISM. A way to test this hypothesis is to look for the abundances of ${ }^{7} \mathrm{Li},{ }^{12} \mathrm{C} /{ }^{13} \mathrm{C}$, and helium in CEMP-no stars. In fact, the wind material is depleted in ${ }^{7} \mathrm{Li}$ and enriched in ${ }^{13} \mathrm{C}$ (see Meynet et al. 2010 and Chiappini et al. 2008).

This work illustrates the importance of future surveys that will enlarge the samples of very metal-poor stars with good abundance determinations. The large statistics brought by these large datasets will enable detailed study not only of the abundance trends but also of the intrinsic scatter in those trends and its metallicity dependence. On the other hand, it is clear that high-quality abundances for key elements such as $\mathrm{CNO}$, the $\mathrm{C}$ isotopic ratio, $\mathrm{n}$-capture elements, and ${ }^{7} \mathrm{Li}$ for a large sample of stars (including CEMP-no stars) is still needed. Spectroscopic follow-ups of metal-poor stars found in the ongoing and planned large surveys (e.g. SEGUE-2 and LAMOST) will play a crucial role in constraining the stellar nucleosynthesis of the first generation of massive stars and in unveiling the role of the fast rotators in the early Universe.

Acknowledgements. G.C. acknowledges financial support from the Fondazione Cassa di Risparmio di Trieste. G.C. and C.C. acknowledge financial support from PRIN2007-MIUR (Italian Ministry of University and Research), Prot.2007JJC53X-001. G.C. and C.C. would like to thank F. Matteucci for useful discussions and suggestions. C.C. acknowledges financial support from the Swiss National Foundation (SNF). C.C. thanks R. Hirschi, S. Ekström, G.Meynet, and M. Limongi for providing the stellar yields and for fruitful discussions.

\section{References}

Aoki, W., Frebel, A., Christlieb, N., et al. 2006, ApJ, 639, 897

Asplund, M., Grevesse, N., \& Sauval, A. J. 2005, ASPC, 336, 25A

Beers, T. C. 2010, in Chemical Abundances in the Universe: Connecting First Stars to Planets, ed. K. Cunha, M. Spite, \& B. Barbuy, in press, IAU Symp. 265

Beers, T. C., \& Christlieb, N. 2005, ARA\&A, 43, 531

Christlieb, N., Bessell, M. S., Beers, T. C., et al. 2002, Nature, 419, 904

Christlieb, N., Gustafsson, B., Korn, A. J., et al. 2004, ApJ, 603, 708

Cayrel, R., Depagne, E., Spite, M., et al. 2004, A\&A, 416, 1117

Cescutti, G. 2008, A\&A, 481, 691

Charbonnel, C., \& Talon, S. 2005, Science, 309, 2189

Chiappini, C., Ekström, S., Meynet, G., et al. 2008, A\&A, 479, L9

Chiappini, C., Hirschi, R., Matteucci, F., et al. 2006, Proceedings of Nuclei in the Cosmos - IX, 80

Chiappini, C., Matteucci, F., \& Ballero, S. K. 2005, A\&A, 437, 429

Chiappini, C., Matteucci, F., \& Meynet, G. 2003, A\&A, 410, 257

Collet, R., Asplund, M., \& Trampedach, R. 2006, ApJ, 644, 121

Ekström, S., Meynet, G., Chiappini, C., Hirschi, R., \& Maeder, A. 2008, A\&A, 489,685

Fabbian, D., Nissen, P. E., Asplund, M., Pettini, M., \& Akerman, C. 2009, A\&A, 500,1143

François, P., Matteucci, F., Cayrel, R., et al. 2004, A\&A, 421, 613

Frebel, A., Aoki, W., Christlieb, N., et al. 2005, Nature, 434, 871

Frebel, A., Christlieb, N., Norris, J. E., et al. 2006, ApJ, 652, 1585

Frebel, A., Collet, R., Eriksson, K., Christlieb, N., \& Aoki, W. 2008, ApJ, 684, 588

Heger, A., Fryer, C. L., Woosley, S. E., Langer, N., \& Hartmann, D. H. 2003, ApJ, 591, 288

Hirschi 2007, A\&A, 461, 571

Israelian, G., Ecuvillon, A., Rebolo, R., et al. 2004, A\&A, 421, 649

Iwamoto, N., Umeda, H., Tominaga, N., Nomoto, K., \& Maeda, K. 2005, Science, 309, 451

Lai, D. K., Bolte, M., Johnson, J. A., et al. 2008, ApJ, 681, 1524

Masseron, T., Johnson, J. A., Plez, B., et al. 2010, A\&A, 509, A93

Matteucci, F., \& Greggio, L. 1986, A\&A, 154, 279

Matteucci, F., \& Recchi, S. 2001, ApJ, 558, 351

Maeder, A., \& Meynet, G. 1989, A\&A, 210, 155

Meynet, G., \& Maeder, A. 2002, A\&A, 390, 561

Meynet, G., Ekström, S., \& Maeder, A. 2006, A\&A, 447, 623

Meynet, G., Hirschi, R., Ekström, S., et al. 2010, Chemical Abundances in the Universe: Connecting First Stars to Planets, ed. K. Cunha, M. Spite, \& B. Barbuy, in press, Review at IAU Symp., 265

Nissen, P. E., Primas, F., Asplund, M., \& Lambert, D. L. 2002, A\&A, 390, 235

Norris, J. E., Christlieb, N., Korn, A. J., et al. 2007, ApJ, 670, 774

Pipino, A., Chiappini, C., Graves, G., \& Matteucci, F. 2009, MNRAS, 396, 1151

Scalo, J. M. 1986, Fund. Cosmic Phys., 11, 1

Schneider, R., Ferrara, A., Natarajan, P., \& Omukai, K. 2002, ApJ, 571, 30

Spite, M., Cayrel, R., Plez, B., et al. 2005, A\&A, 430, 655

Spite, M., Cayrel, R., Hill, V., et al. 2006, A\&A, 455, 291

Woosley, S. E., \& Weaver, T. A. 1995, ApJ, 101, 181 